\title{
High levels of nucleotide diversity and fast decline of linkage disequilibrium in rye (Secale cereale L.) genes involved in frost response
}

\author{
Yongle Li ${ }^{1}$, Grit Haseneyer ${ }^{1}$, Chris-Carolin Schön ${ }^{1}$, Donna Ankerst ${ }^{2}$, Viktor Korzun ${ }^{3}$, Peer Wilde ${ }^{3}$, Eva Bauer ${ }^{\text {** }}$
}

\begin{abstract}
Background: Rye (Secale cereale L.) is the most frost tolerant cereal species. As an outcrossing species, rye exhibits high levels of intraspecific diversity, which makes it well-suited for allele mining in genes involved in the frost responsive network. For investigating genetic diversity and the extent of linkage disequilibrium (LD) we analyzed eleven candidate genes and 37 microsatellite markers in 201 lines from five Eastern and Middle European rye populations.

Results: A total of 147 single nucleotide polymorphisms (SNPs) and nine insertion-deletion polymorphisms were found within 7,639 bp of DNA sequence from eleven candidate genes, resulting in an average SNP frequency of $1 \mathrm{SNP} / 52 \mathrm{bp}$. Nucleotide and haplotype diversity of candidate genes were high with average values $\pi=5.6 \times 10^{-3}$ and $H d=0.59$, respectively. According to an analysis of molecular variance (AMOVA), most of the genetic variation was found between individuals within populations. Haplotype frequencies varied markedly between the candidate genes. ScCbf14, ScVrn1, and ScDhn1 were dominated by a single haplotype, while the other 8 genes (ScCbf2, ScCbf6, ScCbf9b, ScCbf11, ScCbf12, ScCbf15, Sclce2, and ScDhn3) had a more balanced haplotype frequency distribution. Intra-genic LD decayed rapidly, within approximately 520 bp on average. Genome-wide LD based on microsatellites was low.

Conclusions: The Middle European population did not differ substantially from the four Eastern European populations in terms of haplotype frequencies or in the level of nucleotide diversity. The low LD in rye compared to self-pollinating species promises a high resolution in genome-wide association mapping. SNPs discovered in the promoters or coding regions, which attribute to non-synonymous substitutions, are suitable candidates for association mapping.
\end{abstract}

\section{Background}

Rye (Secale cereale L.) is a cross-pollinated cereal with a diploid genome. It is grown on approximately 6 million hectares in Europe for bread-making, animal feed, forage feeding, and vodka production (FAO, 2010). As the most frost tolerant small grain cereal [1] it is well-suited for investigations of frost tolerance. Findings in rye are of interest for less frost tolerant cereals such as wheat and barley.

Cold and frost stress, namely chilling injury at temperatures lower than $10^{\circ} \mathrm{C}$ and freezing injury at temperatures lower than $0^{\circ} \mathrm{C}$, adversely affect plant growth

\footnotetext{
* Correspondence: eva.bauer@wzw.tum.de

${ }^{1}$ Technische Universität München, Plant Breeding, Freising, Germany

Full list of author information is available at the end of the article
}

and productivity via cellular damage, dehydration and metabolic reaction slow-down. A major focus of this study was to investigate candidate genes with a putative role in frost tolerance. Frost tolerance has a polygenic inheritance. Many genes involved in the cold/frost responsive network have been identified in Arabidopsis via quantitative trait loci (QTL) mapping, microarray analysis and transgenic expression [2,3]. These genes are mainly involved in stress signalling, transcriptional regulation, and direct response to cold/frost, including cellular membrane stabilization. The gene $\underline{I}$ ducer of $\underline{C} b f$ Expression 2 (Ice2) is a basic helix-loop-helix transcription factor that binds to promoters of the $\underline{C}$-repeat Binding Factor (Cbf) gene family and activates their transcription under frost stress in hexaploid wheat [4].
C Biomed Central

() 2011 Li et al; licensee BioMed Central Ltd. This is an Open Access article distributed under the terms of the Creative Commons Attribution License (http://creativecommons.org/licenses/by/2.0), which permits unrestricted use, distribution, and reproduction in any medium, provided the original work is properly cited. 
Over-expression of Arabidopsis Ice2 [5] results in increased tolerance to deep freezing stress at a temperature of $-20 C^{\circ}$ after cold acclimation. The $C b f$ gene family belongs to the family of APETALA2 transcription factors. In barley, diploid and hexaploid wheat several cereal Cbf homologs have been cloned and mapped to the Fr2 locus on homoeologous group 5, which coincides with a major QTL for frost tolerance [6-8]. Using wheat-rye addition lines, Campoli et al. [9] assigned twelve members of the $C b f$ gene family to the long arm of chromosome 5R in rye. Several studies in Arabidopsis provide evidence that allelic variation in the $C b f$ gene family forms the molecular basis for the freezing tolerance QTL [10,11]. Cbf transcription factors activate Cold Responsive (COR) genes through binding to cis-elements in the promoters of COR genes under cold stress in Arabidopsis [12]. More than 70 proteins encoded by $C O R$ genes are involved in direct response to cold/frost. Dehydrins, also known as Late Embryogenesis Abundant II (LEA II), are among the proteins that protect other proteins and membranes from cellular damage caused by dehydration [13]. In barley, 13 dehydrin genes ( $D h n$ 1-13) have been identified [14]. Transcripts of Dhn1, Dhn2, Dhn3, Dhn4, Dhn7, and Dhn9 were detected in plants subjected to cold acclimation at $4^{\circ} \mathrm{C}$ followed by mild frost at $-2^{\circ} \mathrm{C}$ or $-4^{\circ} \mathrm{C}$ [15]. Dhn1 and $D h n 3$ were mapped in barley to chromosome $5 \mathrm{H}$ near a QTL for winter hardiness and on chromosome $6 \mathrm{H}$, respectively [13]. Recent studies showed that cold/frost regulation and vernalization are interconnected $[16,17]$. Winter cereals require long exposure to cold in winter, the socalled vernalization, to accelerate flowering in the next spring. This process prevents the early transition of winter cereals into the less cold-tolerant reproductive phase. Vrn1 has been mapped to the second locus conferring frost tolerance, $F r 1$, on the long arm of homoeologous group 5 near the Fr2 locus [18]. Transcript levels of all cold-induced $C b f$ genes at the frost tolerance locus $\mathrm{Fr}-\mathrm{H} 2$ in barley are significantly higher in lines harbouring the $v r n 1$ winter allele than in lines harbouring the $V r n 1$ spring allele [19]. It remains unknown how the $C b f$ family members interact with $V r n 1$ under frost stress.

To unveil genetic diversity among candidate genes involved in the frost response network in rye, one Middle European and four Eastern European populations were studied. Cultivated rye shows a wide range of diversity, reflecting adaptation to various environments and selection pressures [20]. Middle European populations are well-adapted to the more moderate Middle European climate which is in the transition zone between temperate and continental climate, whereas Eastern European populations show good adaptation to a continental climate with severe winters. Thus, differences between Middle and Eastern European populations in allele number and/or frequencies of frost-related candidate genes are expected. Several studies have investigated genome-wide genetic diversity in rye based on molecular markers, including isoenzymes [21] and simple sequence repeats (SSRs) [22]. None, however, have investigated locus-specific genetic diversity at the gene level.

Linkage disequilibrium (LD), the non-random combination of alleles at different loci, determines the marker density required for marker-based studies, such as association mapping or genomic selection [23]. Studies on the extent of LD in various crops, such as Triticum durum [24], Zea mays [25,26], and Sorghum bicolor [27], indicate large variation in the extent of LD. The effect of germplasm on LD is clearly observed in barley, where LD decays within $0.4 \mathrm{~kb}$ in wild material and extends up to $212 \mathrm{~kb}$ in elite lines [28]. LD decay can also vary considerably from locus to locus due to different recombination rates and selection pressures at different regions of the genome. In addition, higher levels of LD are observed in self-pollinating species compared to outcrossing species, indicating that mating systems play a role [23]. Since rye is an outcrossing species, a low level of LD with a rapid decay is expected. To the best of our knowledge there is no prior study on the pattern of LD within and between rye genes.

The objectives of this study were to investigate nucleotide and haplotype diversity, the extent and pattern of LD, and population differences among eleven candidate genes $(S c C b f 2, S c C b f 6, S c C b f 9 b, S c C b f 11$, ScCbf12, ScCbf14, ScCbf15, ScVrn1, ScIce2, ScDhn1, and ScDhn3) involved in the frost tolerance network in five winter rye populations from Belarus, Germany and Poland.

\section{Methods}

\section{Plant material and DNA extraction}

Plant material was derived from five open-pollinated winter rye breeding populations, four from Eastern Europe, PR 2733 (Belarus), EKOAGRO (Poland), SMH2502 (Poland), ROM103 (Poland), and one from Middle Europe, Petkus (Germany). For convenience, they will be referred to as PR, EKO, SMH, ROM, and Petkus, respectively. The Petkus population has undergone several cycles of recurrent selection, while the breeding history of the four Eastern European populations is unknown. Since rye is an outcrossing species, it is highly heterozygous, which leads to difficulties in determining haplotype phase. To address this problem, gamete capture was performed. Between 15 and 68 heterozygous plants from each of the five populations were crossed with the self-fertile inbred line Lo152 resulting in 201 heterozygous $S_{0}$ plants, each with one gamete known. The plants were grown in a growth chamber and DNA 
was extracted from leaves according to Rogowsky et al. [29].

\section{Candidate gene selection and primer design}

Eleven candidate genes, $S c C b f 2, S c C b f 6, S c C b f 9 b$, ScCbf11, ScCbf12, ScCbf14, ScCbf15, ScVrn1, ScIce2, $S c D h n 1$, and ScDhn3, were selected based on their association with frost tolerance in closely related species. Individual $C b f$ genes were selected based on an expression study in rye [30] and linkage mapping in barley and diploid wheat [6,8], $\operatorname{Vrn} 1$ based on linkage mapping and a real-time PCR expression study in wheat $[18,31]$, Ice 2 based on an expression study in wheat [4], and $D h n 1$ and $D h n 3$ based on an expression study in barley [14]. We followed the Cbf nomenclature proposed by Skinner et al. [32], whereby names with the same number followed by different letters describe highly identical but distinct genes, for example, the highly identical $C b f 9 a$ and $C b f 9 b$ genes first identified by Jaglo et al. [33]. Primers for all genes were designed using PrimerBLAST from the NCBI database (http://www.ncbi.nlm. nih.gov/tools/primer-blast/) based on sequences available in GenBank; information can be found in Additional file 1. Due to limited information on rye DNA sequences in GenBank, primers for ScVrn1, ScIce2, $S c D h n 1$ and ScDhn3 were designed based on homologous genes in $H$. vulgare, T. aestivum and T. monococcum. Despite lack of homology in non-coding regions, putative functional regions of the candidate genes could be amplified. A $250 \mathrm{bp}$ fragment of the promoter and first exon of ScVrn1 was amplified since there is evidence that this region is one of the determinants of winter/spring growth habit in barley and wheat $[34,35]$.

\section{Amplification of candidate genes and DNA sequencing}

Fourteen fragments of eleven candidate genes were amplified by PCR in $10 \mu \mathrm{l}$ reaction volumes containing $10 \mathrm{ng}$ DNA, $150 \mathrm{nM}$ of each primer, 1x Taq DNA polymerase reaction buffer, 1.5 or $2.0 \mathrm{mM} \mathrm{MgCl} 2,0.2 \mathrm{mM}$ of each dNTP, and 0.5 U Taq DNA polymerase. After an initial denaturation at $96^{\circ} \mathrm{C}$ for $10 \mathrm{~min}, 35$ cycles were conducted at $96^{\circ} \mathrm{C}$ for $1 \mathrm{~min}$, primer-specific annealing temperatures at $52-66^{\circ} \mathrm{C}$ for $1 \mathrm{~min}, 72^{\circ} \mathrm{C}$ for $1 \mathrm{~min}$, and a final extension step at $72^{\circ} \mathrm{C}$ for $15 \mathrm{~min}$. Details on candidate gene amplification were described in Additional file 1. The PCR products were purified in 96-well MultiScreen PCR plates (Millipore Corporation, Billerica, MA, USA) and directly sequenced through the QIAGEN sequencing service (QIAGEN, Hilden, Germany). Amplicons of each $S_{0}$ plant were sequenced with both forward and reverse PCR primers. Sequence data were assembled into contigs and SNPs were detected using the software Variant Reporter TM V1.0 (Applied Biosystems, Foster City, CA, USA). The DNA sequence of Lo152, a homozygous inbred line, was used as the reference sequence, and alleles of this common parent were subtracted from all sequences to determine the haplotype phase. Heterozygous insertion and deletion events were detected manually by checking sequences from both strands. The web-based program Indelligent v1.2 (http://ctap.inhs.uiuc.edu/dmitriev) was used to resolve heterozygous insertion-deletion events (Indels). In case of large Indels, for example, $200 \mathrm{bp}$ in $S c C b f 2$, which Indelligent could not resolve, amplicons from the respective lines were sub-cloned using the TOPO TA Cloning Kit (Invitrogen, Carlsbad, CA, USA). At least five clones were sequenced to resolve heterozygous Indels. Sequences of the Lo152 reference alleles from the eleven candidate genes were submitted to GenBank under accession numbers HQ730763-HQ730773.

The actual numbers of successful PCR amplification of the 201 lines differed from gene to gene ranging from 128 lines (64\%) in ScCbf11 to 198 (98\%) in ScVrn1. Missing amplification products in individual lines were most likely the result of SNPs/Indels in the primer binding sites. However, absence of some Cbf genes in particular lines, as has recently been reported in barley and wheat $[36,37]$ cannot be excluded as an alternative explanation.

\section{Sequence analysis}

Sequence polymorphisms were deduced from sequence comparisons in gene-wise sequence alignments. For convenience, polymorphic sites along the sequence were numbered starting with "SNP1". Lo152 alleles were excluded from all analyses. Haplotypes and haplotype frequencies were determined within each candidate gene using DnaSP v5.10 [39] and Arlequin v3.1 [40], respectively.

Nucleotide diversity $(\pi)$ was calculated as the average number of nucleotide differences per site between two sequences for both, the complete sequences and restricted to exons, and haplotype diversity $(H d)$ as the probability that two randomly chosen haplotypes from a given population were different [37]. Analyses of nucleotide and haplotype diversity were performed separately for each population as well as for all populations grouped together using the software DnaSP v5.10. DnaSP v5.10 does not take into account alignment gaps that may lead to underestimated diversity values. Hence, to avoid potential bias, Indels were treated as single polymorphic sites. Average nucleotide diversity $(\pi)$ over all genes was calculated using concatenated sequences in software TASSEL v2.1 (http://www.maizegenetics.net/).

To test for selection Tajima's $D$ was calculated as the difference between the mean pairwise nucleotide differences $(\pi)$ and the number of segregating sites $(S)$ relative to their standard error using the software DnaSP v5.10. The statistical significance of Tajima's $D$ was 
obtained assuming that $D$ follows the beta distribution [38]. The rate ratio of non-synonymous to synonymous substitutions $\left(d_{N} / d_{S}\right)$ was calculated according to the method introduced by Yang and Nielsen [41] implemented in the program YN00 of software package PAML v4.4c [38]. Significant departure from the standard neutral model, i.e. $d_{N} / d_{S}=1$, was assessed by the likelihood ratio test implemented in the CODEML program of PAML v4.4c.

\section{SSR genotyping and genetic diversity analyses}

Thirty seven SSR markers were chosen based on their experimental quality and map location as providing comprehensive coverage of the rye genome. Primers and PCR conditions for rye microsatellite (RMS) and Secale cereale microsatellite (SCM) markers were described in detail by Khlestkina et al. [39] and Hackauf and Wehling [40], respectively. Fragments were separated using a 3130xl Genetic Analyzer (Applied Biosystems Inc., Foster City, CA, USA), and allele sizes were assigned using the program GENEMAPPER (Applied Biosystems Inc., Foster City, CA, USA). Genotyping data obtained from the SSR analyses of the 201 lines were used for the following calculations. Polymorphic information content (PIC) was estimated using PowerMarker v3.0 [41], and 95\% confidence intervals were calculated based on 10,000 bootstrap replications. To eliminate bias whereby the observed number of alleles highly depends on the number of analysed genotypes, allelic richness $(R s)$ was estimated from a rarefaction method [42] implemented in Fstat v2.9.3 [43]. Briefly, the method estimates the expected number of alleles in a sub-sample of $n$ genotypes, given that $N$ genotypes have been sampled at a locus, where $\mathrm{N} \geq \mathrm{n}$. Specifically, in this study, it was calculated as

$$
R_{s}=\sum_{i=1}^{s}\left[1-\frac{\left(\begin{array}{c}
N-N_{i} \\
n
\end{array}\right)}{\left(\begin{array}{c}
N \\
n
\end{array}\right)}\right]
$$

where $N$ was the number of observed genotypes (201 or less), $N_{i}$ the number of genotypes with type $i$ alleles among the $N$ genotypes, $n$ the number of genotypes in each population, and $S$ was the total number of alleles among the $N$ genotypes. To visualize the degree of variation within and between populations, principal coordinate analysis (PCoA) was performed using NTSYSpc v2.2 (Applied Biostatistics Inc., Setauket, NY, USA) based on DICE similarity coefficients for SSRs and haplotypes of candidate genes [44]. Analysis of molecular variance (AMOVA) [45] was performed based on SSRs using Arlequin v3.1 [46] with 15,000 permutations of the data to estimate statistical significance at $P<0.001$ for each variance component in Fisher's exact test. The Lo152 alleles were excluded from all analyses.

\section{Linkage disequilibrium}

Linkage disequilibrium was measured by the parameter $r^{2}$ [47] for candidate genes and SSR markers using DnaSP v5.10 and TASSEL v2.1, respectively, with Indels treated as single polymorphic sites and SNPs with minor allele frequencies (MAF) $<0.05$ excluded due to instability. Statistical significance of LD was calculated using Fisher's exact test [48] and decay examined exploratorily by graphs of pairwise distances (bp) versus $r^{2}$. Under the mutation-drift-equilibrium model, the expected value of $r^{2}$ is

$$
\mathrm{E}\left(r^{2}\right)=1 /(1+4 N c)
$$

where $N$ is the effective population size, and $c$ is the recombination fraction between sites. With assumption of a low mutation rate and an adjustment for sample size, the expectation becomes [49]:

$$
E\left(r^{2}\right)=\left[\frac{10+\Gamma}{(2+\Gamma)(11+\Gamma)}\right]\left[1+\frac{(3+\Gamma)\left(12+12 \Gamma+\Gamma^{2}\right)}{n(2+\Gamma)(11+\Gamma)}\right]
$$

where $\Gamma=4 N c$ and $n$ is the number of lines compared. The LD decay curve was estimated using a nonlinear least-squares estimate of $\Gamma$ fit by the $n l s$ function in the $\mathrm{R}$ software package, http://www.r-project.org, separately for each population and for all populations pooled together. The approach of Breseghello and Sorrells [50] was used to determine threshold values of $r^{2}$ that indicated significant LD. Briefly, $r^{2}$ values were estimated from 37 unlinked SSR markers and square root transformed so that they would be better approximated by a Normal distribution. The 95 th percentile from the empirical distribution of all pairwise $r(\mathrm{n}=666)$ derived from the 37 unlinked SSR markers was selected as the threshold value, with the rationale that any values above the threshold could in high likelihood be attributable to genetic linkage. Threshold values were calculated separately for each population and for all populations pooled together. The extent of LD was estimated as the point where the LD decay curve passed below the threshold.

\section{Results}

\section{DNA sequence polymorphisms}

In total, 7,639 bp from eleven candidate genes in 201 rye lines were amplified resulting in 147 SNPs, nine Indels, and an average SNP frequency of $1 \mathrm{SNP} / 52 \mathrm{bp}$ (Table 1). Thirty nine SNPs were non-synonymous polymorphisms resulting in amino acid replacements, 15 of which changed polarity. In the $C b f$ gene family, $S c C b f 9 b$ 
Table 1 Summary information of candidate gene (CG) sequences: Analyzed fragment length, gene coverage, number of lines, number of SNPs, rate ratio of non-synonymous to synonymous substitutions $\left(d_{N} / d_{s}\right)$, number of Indels and haplotypes, haplotype (Hd) and nucleotide diversity $(\pi)$, Tajima's $D$, and linkage disequilibrium (LD)

\begin{tabular}{|c|c|c|c|c|c|c|c|c|c|c|c|}
\hline$\overline{C G}$ & $\begin{array}{l}\text { Fragment length } \\
\text { (bp) }\end{array}$ & $\begin{array}{l}\text { Gene } \\
\text { coverage }\end{array}$ & $\begin{array}{l}\text { No. of } \\
\text { lines b }\end{array}$ & $\begin{array}{l}\text { No. of SNPs c } \\
\text { (non-synonymous) }\end{array}$ & $d_{N} / d_{S}$ & $\begin{array}{l}\text { No. of } \\
\text { Indels }\end{array}$ & $\begin{array}{l}\text { No. of } \\
\text { haplotypes }\end{array}$ & $H d \pm S D$ & $\begin{array}{l}\pi \pm S D \times 10^{-3} \\
\text { (only exon) }\end{array}$ & Tajima's $D$ & $\begin{array}{l}\text { Intra-genic LD } \\
\left(r^{2}\right)\end{array}$ \\
\hline ScCbf2 & 619 & $5^{\prime} U T R / E$ & 169 & $2(0)$ & 0.001 & 1 & 7 & $\begin{array}{l}0.67 \pm \\
0.02\end{array}$ & $\begin{array}{l}1.5 \pm 0.1(1.4 \pm \\
0.1)\end{array}$ & 1.17 & 0.13 \\
\hline ScCbf6 & 495 & E & 197 & $3(0)$ & 0.023 & 0 & 9 & $\begin{array}{l}0.44 \pm \\
0.04\end{array}$ & $3.6 \pm 0.3$ & -0.35 & 0.77 \\
\hline scCbfob & 1,371 & 5'UTR/E/3'UTR & 183 & $30(10)$ & $0.174^{* * *}$ & 1 & 95 & $\begin{array}{l}0.98 \pm \\
0.03\end{array}$ & $\begin{array}{l}7.1 \pm 0.3(11.5 \pm \\
0.2)\end{array}$ & 1.71 & 0.14 \\
\hline ScCbf11 & 623 & E & 128 & $27(12)$ & 0.165 & 0 & 12 & $\begin{array}{l}0.65 \pm \\
0.02\end{array}$ & $14.5 \pm 0.9$ & 1.74 & 0.51 \\
\hline ScCbf12 & 754 & 5'UTR/E/3'UTR & 141 & $25(8)$ & $0.286^{* * *}$ & 1 & 48 & $\begin{array}{l}0.89 \pm \\
0.02\end{array}$ & $\begin{array}{l}8.8 \pm 1.0(7.7 \pm \\
0.1)\end{array}$ & 0.40 & 0.38 \\
\hline ScCbf14 & 560 & E & 185 & $5(3)$ & $0.606^{* *}$ & 0 & 4 & $\begin{array}{l}0.17 \pm \\
0.04\end{array}$ & $1.5 \pm 0.3$ & -0.27 & 0.92 \\
\hline ScCbf15 & 502 & E & 172 & $3(3)$ & $1.490^{* * *}$ & 1 & 9 & $\begin{array}{l}0.68 \pm \\
0.04\end{array}$ & $3.0 \pm 0.2$ & $2.14^{*}$ & 0.30 \\
\hline ScDhn1 & 435 & $5^{\prime} U T R / E$ & 138 & $4(1)$ & $0.128^{* *}$ & 2 & 12 & $\begin{array}{l}0.33 \pm \\
0.05\end{array}$ & $\begin{array}{l}2.7 \pm 0.5(4.4 \pm \\
0.1)\end{array}$ & $-1.86^{*}$ & 0.48 \\
\hline ScDhn3 & 514 & I/E/3'UTR & 130 & $12(2)$ & $0.229^{* * *}$ & 2 & 21 & $\begin{array}{l}0.73 \pm \\
0.03\end{array}$ & $\begin{array}{l}8.1 \pm 0.6(8.9 \pm \\
0.1)\end{array}$ & 0.008 & 0.25 \\
\hline Sclce2 & 1,224 & I/E & 189 & $36^{d}$ & n.a. & 0 & 32 & $\begin{array}{l}0.80 \pm \\
0.02\end{array}$ & $11.2 \pm 0.6(0)$ & $2.34^{*}$ & 0.36 \\
\hline ScVrn1 & 542 & $5^{\prime} U T R / E$ & 198 & 0 & n.a. & 1 & 2 & $\begin{array}{l}0.11 \pm \\
0.03\end{array}$ & $0.4 \pm 0.1(0)$ & -0.33 & n.a. \\
\hline Total & 7,639 & & & 147 (39) & & 9 & 251 & & & & \\
\hline
\end{tabular}

${ }^{\mathrm{a}}$ E: exon; UTR: untranslated region; l: intron.

${ }^{b}$ Failure of amplification in some of the lines may be due to the presence of SNPs/Indels in the binding sites of the sequences and/or the absence of some of the Cbf genes in some particular lines.

c Minor allele frequency (MAF) $>0.05$.

${ }^{\mathrm{d}}$ SNPs are silent since they were all located in the first intron of the gene.

Significance levels: $* P<0.05$, * $P<0.01, * * * 0.001$.

n.a.: not available. 
had the highest number of SNPs $(\mathrm{N}=30)$, of which ten were non-synonymous and three led to an exchange of amino acids of different polarity. The first intron and second exon comprising $20 \%$ of the coding sequence of ScIce 2 were amplified, resulting in the identification of 36 SNPs, all located in the first intron. A 250 bp fragment of the promoter and first exon of ScVrn1 was amplified but no polymorphic site was identified, except for a 2 bp Indel. Out of nine Indels identified, seven were located in the non-coding regions of $S c C b f 2$, ScCbf9b, ScVrn1, ScDhn1, and ScDhn3 and two in the coding regions of $S c C b f 12$ and $S c C b f 15$ without causing a frame shift (Table 1). It is noteworthy that the $200 \mathrm{bp}$ Indel in the promoter of $S c C b f 2$ contained two MYB and one MYC cis-elements, putative binding sites for the transcription factor ScIce2.

\section{Locus-wise and genome-wide genetic diversity}

Nucleotide diversity $(\pi)$ ranged from $0.4 \times 10^{-3}$ in $S c V r n 1$ to $14.5 \times 10^{-3}$ in $S c C b f 11$, and when restricted to exons, from 0 in ScIce 2 and ScVrn1 to $14.5 \times 10^{-3}$ in ScCbf11 (Table 1). The biggest difference between analyses of $\pi$ for the whole gene compared to restriction to exons occurred in ScIce 2 where $\pi$ decreased from 11.2 to 0 due to absence of SNPs in the exon. Haplotype diversity $(H d)$ ranged from 0.11 in $S c V r n 1$ to 0.98 in $S c C b f 9 b$. A significant positive Tajima's $D$ value was observed over all populations for ScCbf15 and ScIce2, whereas a significant negative value was observed in ScDhn1. Rate ratios of non-synonymous to synonymous substitutions $\left(d_{N} / d_{S}\right)$ were $<1$ for $S c C b f 2, S c C b f 6$, ScCbf9b, ScCbf11, ScCbf12,ScCbf14,ScDhn1, and $S c D h n 3$. ScCbf15 was the only gene with a $d_{N} / d_{S}$ ratio $>1 . d_{N} / d_{S}$ was significant for $S c C b f 9 b, S c C b f 12$, ScCbf14, ScCbf15, ScDhn1, and ScDhn3. Due to lack of polymorphisms in their coding sequences $d_{N} / d_{S}$ was not calculated for ScIce2 and ScVrn1.

In the SMH population, ScCbf6, ScIce2, and ScDhn1 had reduced nucleotide and haplotype diversities. Similarly in the PR and EKO populations, respectively, $S c C b f 11$ and ScCbf15 had reduced nucleotide and haplotype diversities compared to the other genes (Additional file 2). Haplotype frequencies varied markedly between candidate genes, with some candidate genes dominated by a single haplotype and others with a more balanced haplotype frequency distribution (Figure 1). For example, in ScCbf14, ScVrn1, and ScDhn1, the most frequent haplotype occurred in more than $70 \%$ of genotypes, whereas in $S c C b f 9 b$ all haplotypes occurred with frequencies less than $10 \%$. The finding in $S c C b f 9 b$ can be attributed to a large number of haplotypes $(\mathrm{N}=95)$ with high haplotype diversity primarily generated by polymorphic sites located in the coding region. Similarly, only five of 48 haplotypes in ScCbf12 occurred at a frequency greater than $10 \%$. For $S c C b f 14$, all populations had a similar distribution of haplotype frequencies. However, for $S c C b f 15$ haplotypes $1,2,3$, and 4 were evenly distributed in PR, whereas in the other four populations only two haplotypes (EKO and SMH: 1 and 2; ROM and Petkus: 1 and 4) were prevalent (80\% $95 \%)$. For $S c C b f 11$, haplotype 1 was predominant in the PR and Petkus populations, occurring in $82 \%$ and $57 \%$ of lines, respectively, whereas haplotype 2 predominated in EKO (67\%) and SMH (75\%).

Genetic diversity within the five populations was summarised based on 37 genome-wide SSR markers (Table 2). A total of 230 alleles and an average of 6.2 alleles per locus were observed. PIC varied from $0.37 \pm$ 0.02 to $0.51 \pm 001$ with an average of 0.47 . Allelic richness, which is not affected by sample size, ranged from 2.51 to 3.43 , with a mean of 3.16. PIC was highly correlated with allelic richness $(r=0.965)$. Compared to the four Eastern European populations, the Petkus population had a slightly lower mean number of alleles per locus, PIC, allelic richness and number of private alleles, despite the fact that it had the largest population size. Genetic diversities of individual SSR markers across the five populations are provided in Additional file 3.

\section{Genetic variation within and between populations}

PCoA of candidate gene haplotypes revealed large genetic variation within each population and no clustering according to population membership (Figure 2). The first and second principal co-ordinates explained 10.3\% and $9.7 \%$ of the total genetic variation, respectively. PCoA of the 37 genome-wide SSRs similarly identified most genetic variation as residing within populations (Figure 3). However, it could differentiate the Petkus population from all Eastern European populations, and the PR population from the other three Eastern European ones. The first and second principal co-ordinates explained $7.3 \%$ and $4.1 \%$ of the total genetic variation, respectively. AMOVA revealed low variation (13.3\%) between populations, but high variation $(86.7 \%)$ within populations (Additional file 4).

\section{Linkage disequilibrium}

The mean $r^{2}$ for pairs of SNPs within candidate genes ranged from 0.13 to 0.92 (Table 1 ). Two strong LD blocks were observed, one in the coding sequence of $S c C b f 14$ and one in the promoter region of $S c C b f 9 b$, with mean $r^{2}$ values 0.92 and 0.85 within the two LD blocks, respectively (Figure 4). In ScCbf11, two strong LD blocks were observed, one in the interval from SNP1 to SNP12 spanning 99 bp (mean $r^{2}$ within LD block = 0.93), and one from SNP17 to SNP27, spanning $243 \mathrm{bp}$ (mean $r^{2}$ within LD block $\left.=0.98\right)$. On the contrary, low LD was observed in ScCbf2 (mean $r^{2}=0.13$ ), ScDhn3 


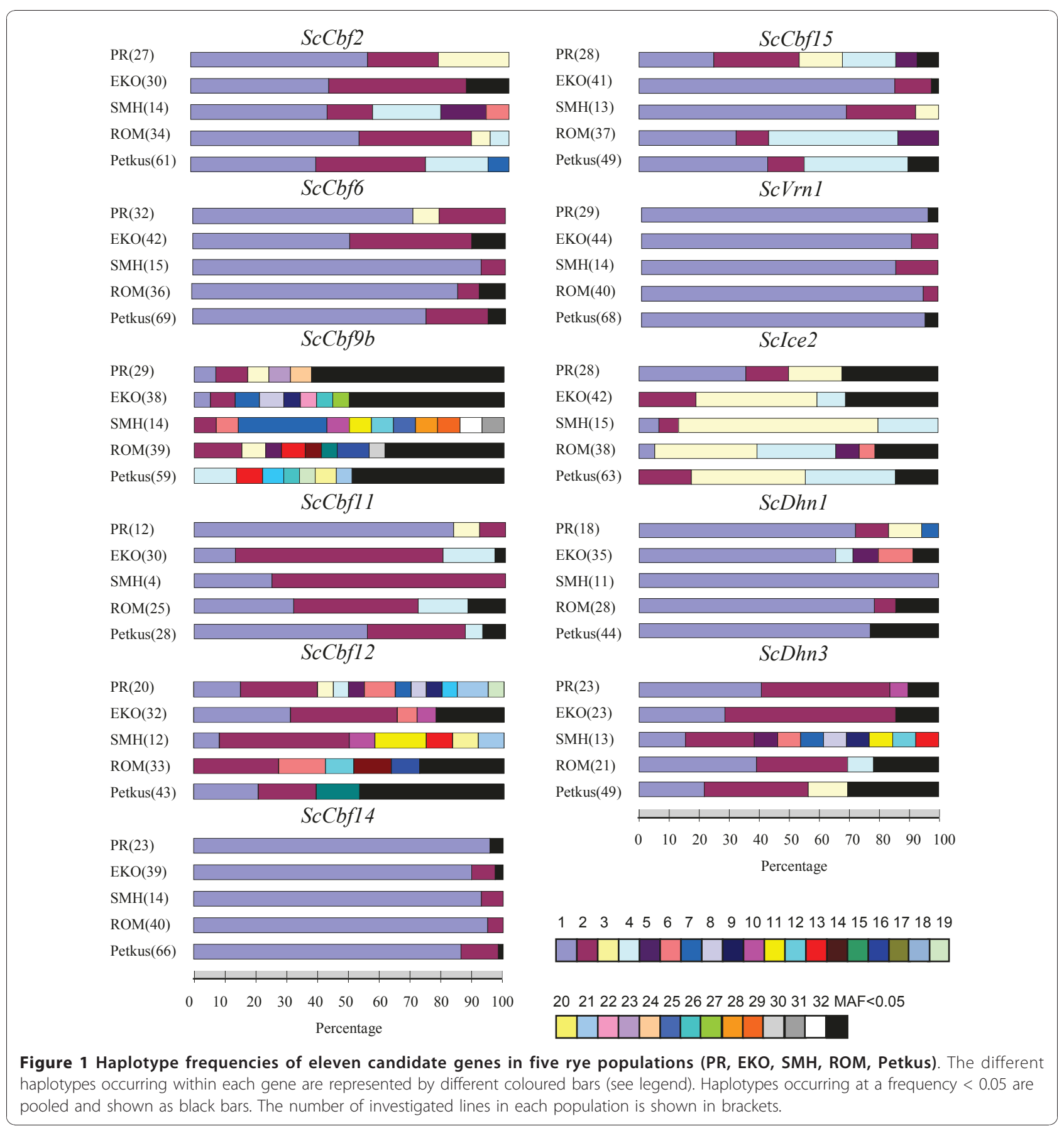

(mean $r^{2}=0.25$ ) and in the coding sequence of $S c C b f 9 b$ (mean $r^{2}=0.14$ ). Estimation of LD in ScIce 2 was performed based on 36 SNPs (mean $r^{2}=0.36$ ), all located in the first intron of the gene. There were three strong LD blocks, from SNP1 to SNP18 (block 1), SNP19 to SNP31 (block 2), and SNP32 to SNP36 (block 3), spanning $458 \mathrm{bp}, 187 \mathrm{bp}$, and $61 \mathrm{bp}$, with a mean $r^{2}$ within LD blocks of $0.85,0.75$, and 0.73 , respectively. Interestingly, the mean $r^{2}$ between blocks 2 and 3 decreased to
0.35 , between blocks 1 and 2 , further to 0.10 , and between blocks 1 and 3, to 0.13. The inter-genic LD among the $S c C b f$ genes was very low (mean $\left.r^{2}=0.05\right)$, and only ScCbf14 showed a slightly higher LD (mean $r^{2}$ $=0.15$ ) than $S c C b f 9 b$ (data not shown). Threshold values of $r^{2}$ as determined from 37 unlinked SSR markers varied from 0.16 over all populations to 0.46 in the $\mathrm{SMH}$ population. The average extent of significant LD pooling all candidate genes and populations together was 
Table 2 Genetic diversity within populations based on 37 SSR markers

\begin{tabular}{|c|c|c|c|c|c|}
\hline Population & No. of lines & No. of private alleles ${ }^{a}(\%)$ & Average no. of alleles (range) & $\mathrm{PIC}^{\mathrm{b}} \pm \mathrm{SD}$ & Allelic richness $^{c}$ \\
\hline PR & 33 & $20(12.1 \%)$ & $4.46(2-12)$ & $0.50 \pm 0.02$ & 3.43 \\
\hline EKO & 44 & $14(8.8 \%)$ & $4.30(2-18)$ & $0.49 \pm 0.03$ & 3.28 \\
\hline $\mathrm{SMH}$ & 15 & $3(2.4 \%)$ & $3.38(1-9)$ & $0.46 \pm 0.03$ & 3.18 \\
\hline $\mathrm{ROM}$ & 41 & $13(7.7 \%)$ & $4.50(2-13)$ & $0.51 \pm 0.01$ & 3.38 \\
\hline Petkus & 68 & $4(3.6 \%)$ & $3.00(1-10)$ & $0.37 \pm 0.02$ & 2.51 \\
\hline Mean & & 10.80 & 3.93 & 0.47 & 3.16 \\
\hline
\end{tabular}

a Private alleles denotes the number of alleles which occurred only in one population.

b PIC: Polymorphic information content, a higher value means higher genetic diversity.

c Allelic richness is a measure of the number of alleles independent of sample size, a higher value means higher genetic diversity.

approximately 520 bp (Figure 5). There were 2,194 pairwise comparisons of polymorphic sites, of which almost one third were significant as determined by Fisher's exact test. The average extent of significant LD in individual populations was much smaller because of more stringent threshold values and ranged from 0 to approximately $380 \mathrm{bp}$ in the SMH and Petkus populations, respectively. Extent of LD ranged from approximately 80 bp in ScCbf15 to 800 bp in ScIce2 (Additional file 5). In ScCbf11, ScCbf14, and ScDhn1, mean $r^{2}$ remained larger than 0.16 within the $400 \mathrm{bp}$ amplified region. As expected LD based on genome-wide SSR markers was low with a mean $r^{2}=0.01$ (data not shown).

\section{Discussion}

High level of nucleotide and haplotype diversity in rye We investigated the genetic diversity of five winter rye populations from Middle and Eastern Europe. SNP frequency and nucleotide diversity are affected by several

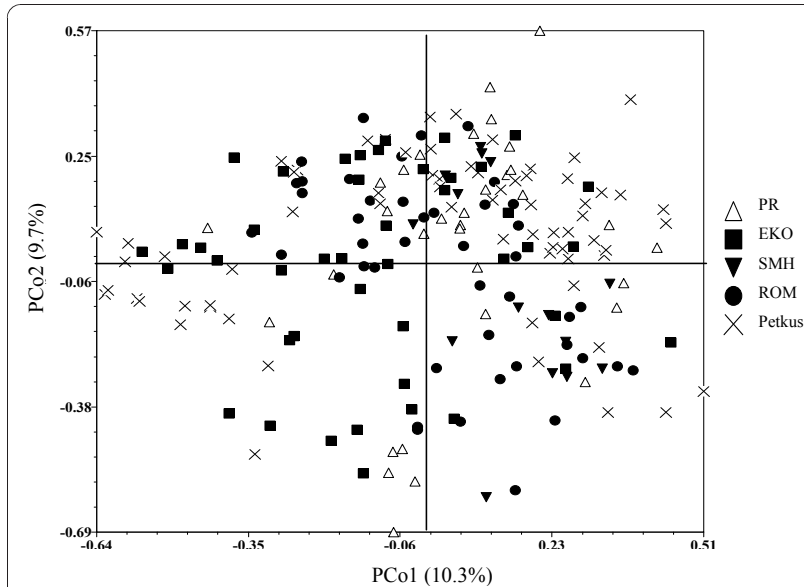

Figure 2 Principal co-ordinate analysis of 201 rye lines from five populations (PR, EKO, SMH, ROM, Petkus) based on candidate gene haplotypes. Analysis was based on a similarity matrix of candidate gene haplotypes. PCo1 and PCo2 are the first and second principal co-ordinates and percentages indicate percent variation explained. factors, including selection, mutation, mating system, effective population size, and demography [51]. SNP frequency observed in the 5 rye populations under study was on average 1 SNP every $52 \mathrm{bp}$ and the average nucleotide diversity $(\pi)$ ranged from $0.4 \times 10^{-3}$ to $14.5 \times$ $10^{-3}$ with an average value of $\pi=5.6 \times 10^{-3}$. These values are as high as those reported in maize landraces, where one study reported a rate of one SNP per $62 \mathrm{bp}$, a range of $\pi$ from $0.1 \times 10^{-3}$ to $13.3 \times 10^{-3}$ and an average value of $\pi$ equal to $4.0 \times 10^{-3}$ [52]. Some studies have suggested that comparisons among different species should be restricted to homologous genes [53]. Nucleotide diversities of three Cbf homologs (AtCbf1, AtCbf2 and AtCbf3) in 34 Arabidopsis ecotypes ranged from $\pi=2.6 \times 10^{-3}$ to $6.9 \times 10^{-3}$ [54], a smaller range compared to this study $\left(\pi=1.5 \times 10^{-3}\right.$ to $\left.14.5 \times 10^{-3}\right)$, which is likely due to the different mating system. In addition, the $C b f$ gene family in rye encompasses more members than in Arabidopsis, which could result in less selection pressure on individual genes with

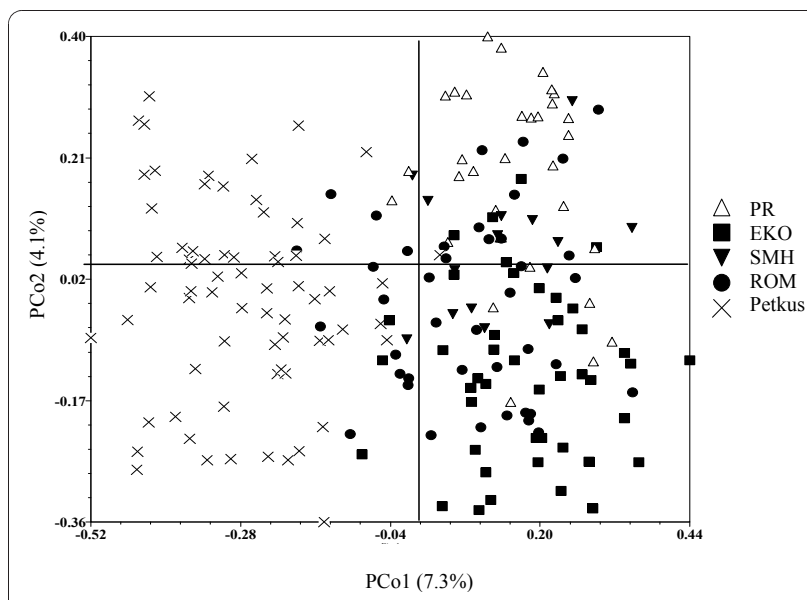

Figure 3 Principal co-ordinate analysis of 201 rye lines from five populations (PR, EKO, SMH, ROM, Petkus) based on genome-wide SSR markers. Analysis was based on a similarity matrix from 37 SSR loci. PCo1 and PCo2 are the first and second principal co-ordinates and percentages indicate percent variation explained. 

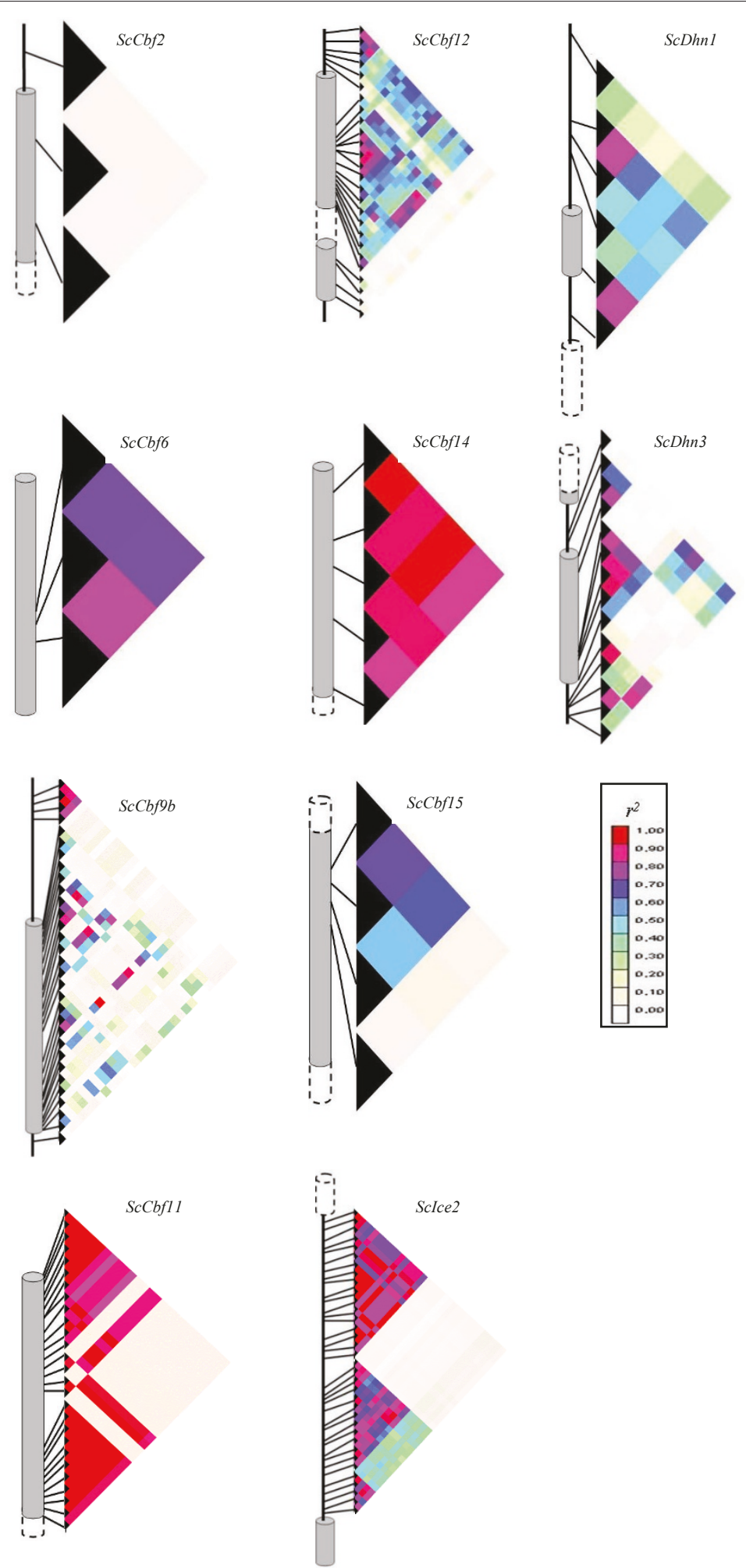

Figure 4 LD heat plots of ten candidate genes. Analysed sequences, including the promoter and complete coding sequences of $S c C b f 6$ and ScCbf9b, and partial coding sequences of ScCbf12, ScCbf14, and ScCbf15; ScVrn1 was not included due to a lack of pairwise comparisons, since only one Indel was observed. Exons, and 5'- or 3'-flanking regions are represented by grey cylinders and black lines, respectively. White cylinders with dashed lines indicate non-amplified exons. Black triangles represent polymorphic sites starting from "SNP1" on the top of each graph. Each grid represents the strength of LD estimated by $r^{2}$ for each pairwise comparison between polymorphic sites with a minor allele frequency (MAF) $>0.05$. The colour legend for $r^{2}$ values is given on the right side. 


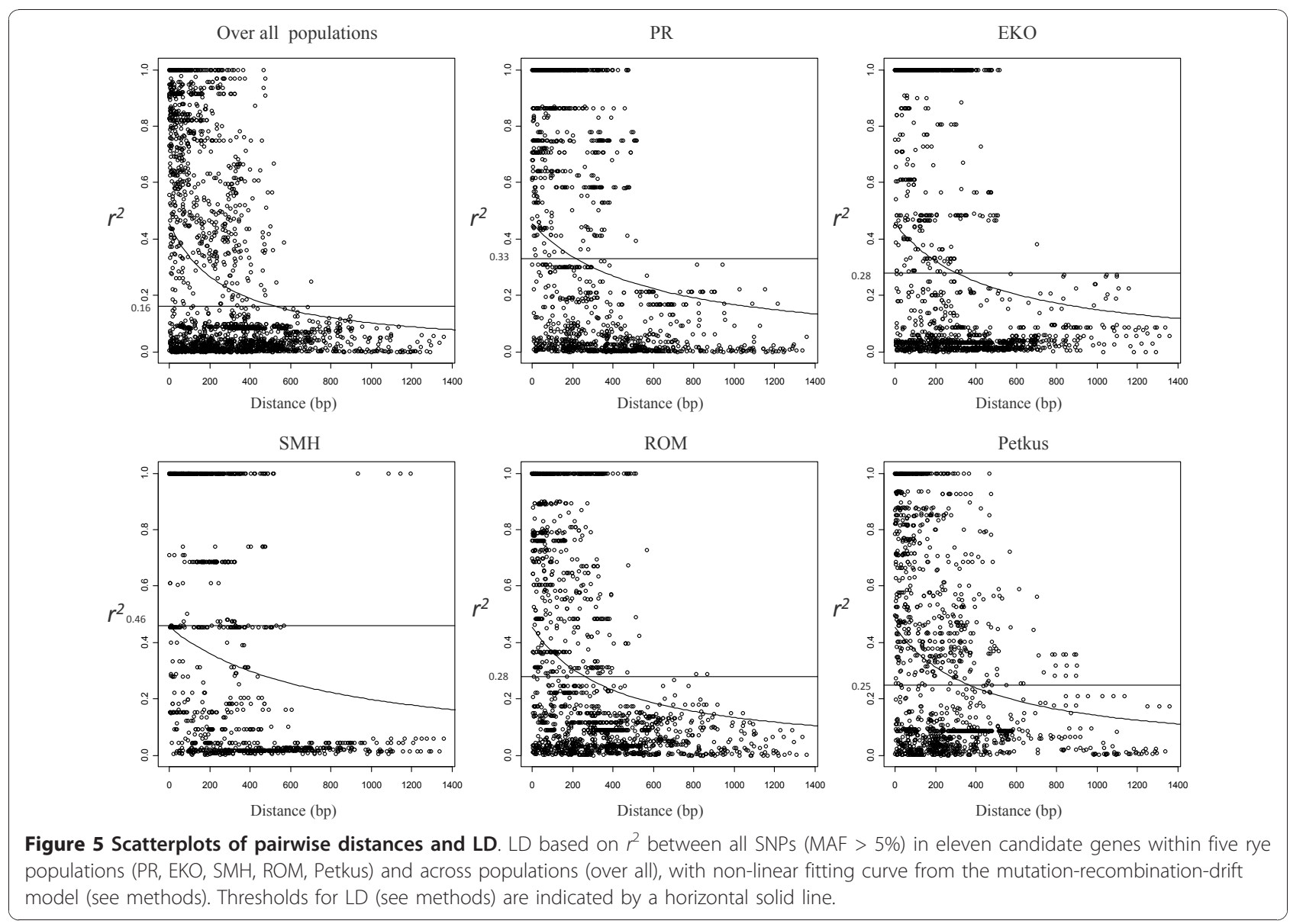

complementary function in the frost tolerance network and consequently in a higher nucleotide diversity. The buffering effect induced by a large number of duplicated genes leads to a higher variation in individual duplicated genes, a phenomenon also observed in polyploid plants [55]. It is worth re-iterating that inference concerning the nucleotide diversity of ScVrn1 was restrained since only a partial fragment of the gene, $30 \%$ of the coding region, could be amplified due to limited available rye sequences for primer design. Observed haplotype diversities of $H v C b f 9 b$ in Hordeum spontaneum, old cultivars and modern cultivars of $H$. vulgare were $0.48,0.18$, and 0.06 , respectively, which is much lower than that of $S c C b f 9 b$ in this study $(0.98 \pm 0.03)$ [36].

\section{Directional selection}

A reduced genetic diversity was observed in five of the eleven genes. One possible explanation is that directional selection on the loci responsible for fitness related traits such as frost tolerance might reduce diversity within locally adapted populations due to an increase in the frequency of alleles contributing to adaptation [56].
ScCbf15 and ScIce2 showed significant positive values of Tajima's $D(2.14$ and 2.34, respectively; $P<0.05)$ over all populations, indicating balancing selection, whereby genotypes carrying alleles with intermediate frequency are favored. Positive Tajima's $D$ values can also be observed if a population was formed from a recent admixture of two different populations, which cannot be excluded in this study. Dhn1 showed a significant negative value of Tajima's $D(P<0.05)$, indicating purifying selection, whereby an excess of polymorphisms with low frequencies was observed. However, population growth can also result in significant negative values of Tajima's D. Interestingly, Dhn1 in Scots pine has also been described as subject to positive selection [57], implying that $D h n 1$ is possibly a target of selection in different species. ScCbf9b, ScCbf12, ScCbf14, ScDhn1, and ScDhn3 had a $d_{N} / d_{S}$ ratio significantly smaller than $1(P<0.01$ or $P<0.001)$, whereas $S c C b f 15$ had a $d_{N} / d_{S}$ ratio significantly greater than $1(P<0.001)$. These findings can be interpreted as indication for purifying and positive selection, respectively [58]. However, it was pointed out that inferring selection pressure based on the $d_{N} / d_{S}$ ratio is difficult from within-species data where segregating 
polymorphisms rather than fixed substitutions are observed [53,58,59].

Based on haplotype frequencies in the eleven candidate genes, the single Middle European population did not distinctly differ from the Eastern European ones (Figure 1). Since we have no information on the temporal breeding history of the Eastern European populations, it is beyond the scope of this study to make inferences on the selection pressure due to contrasting winter temperatures in these Middle and Eastern European populations. One possible explanation for a lack of differentiation might be seed exchanges between them. However, little is known about these processes, since pedigrees of the four Eastern European populations were not accessible.

\section{Genetic variation within and among populations}

Assessment of genetic diversity based on genome-wide SSRs and locus-specific candidate genes are complementary investigations, the former providing a global view of the rye genome and the latter restricted to genes involved in the frost tolerance network. Genome-wide assessment of diversity using SSR markers revealed a higher genetic diversity for the Eastern European populations PR, EKO, SMH, and ROM compared to the Middle European Petkus population. One reason for this finding might be a bottleneck effect due to a higher selection pressure in the Petkus population, whereby it could be assumed that many "unfavourable" minor alleles were eliminated to pave the way for plants with desirable traits. The Petkus population, one of the major heterotic groups in rye, has systematically been improved by more than 5 cycles of full sib recurrent selection and a reduction in allele diversity of SSR markers due to hitchhiking with linked loci which were targets of selection is probable. The reduction of genetic diversity due to human-induced selection has been well documented in barley and maize [36,60,61]. By contrast, the Eastern European populations experienced a lower selection pressure by mass or half sib selection in the breeding programs, where introgression of foreign material was common in order to keep genetic variability on a high level. Interestingly, no reduction of genetic diversity was observed in the Petkus population based on candidate genes. One possible explanation is that at the time where selection took place, winters in Germany, the provenance of the Petkus population, were harsh enough to form a similar selection pressure on the Petkus population compared to Eastern European populations under Eastern European winters. It must be stated however, that Petkus is the only representative for the Middle European rye populations in our study and thus our conclusions on population differences must be limited to the Petkus population. Another reason could be that frost tolerance is a complex quantitative trait involving large gene networks comprising individual genes contributing only small effects, thereby making it difficult to detect selection signatures, such as reduction of genetic diversity in candidate genes. PCoA based on both candidate genes and SSRs showed high genetic variation between individuals within populations and limited clustering of lines from the same population, findings in accordance with previously reported investigations of 26 rye populations based on isoenzyme markers [21] and 12 rye populations based on RFLP markers [21]. Similar results have also been reported in other outcrossing species, including white clover [62] and perennial ryegrass [63], probably a consequence of the obligate cross-pollinated reproductive behaviour of outcrossing species. On the contrary, investigations in the self-pollinated species rice have revealed larger variation between populations [64].

\section{Rapid decay of linkage disequilibrium in rye}

The extent of LD in rye across all eleven candidate genes and over all populations was approximately 520 bp using $r^{2}=0.16$ as a critical threshold estimated from a separate analysis of 37 unlinked SSR markers. This rapid decay of LD could be expected, because compared to self-pollinated species, cross-pollinated rye has a higher effective recombination rate, which leads to a rapid decay of LD [23]. LD decays rapidly in other cross-pollinated species, including douglas fir, maize and ryegrass $[25,53,65]$. However, in self-pollinated species LD can extend up to 10-30 kb as in Arabidopsis [66,67] and $212 \mathrm{~kb}$ in cultivated barley [28]. Pairwise LD measured by $r^{2}$ based on SSRs was very low (mean $r^{2}=$ 0.01), which was expected since the 37 SSRs have an average marker interval of $21 \mathrm{cM}$ according to the integrated consensus map of Gustafson et al. [68].

LD results from the interplay of many factors. Selection, which causes locus-specific bottlenecks, is one of the factors that increases LD between selected alleles at linked loci. Homologs of $S c C b f$ (except $S c C b f 11$ in this case) were closely linked and located in the $\mathrm{Fr}-\mathrm{H} 2 / \mathrm{Fr}$ $A^{m} 2$ frost locus spanning approximately $0.8 \mathrm{cM}$ in the genetic maps of barley and diploid wheat on homoeologous group $5[6,8,17]$. The order of $C b f$ genes in the genetic map is consistent in both species [17]. The Cbf gene family is a large regulatory gene family with more than 20 members in barley, diploid and hexaploid wheat $[6,8,69]$, sharing a high sequence similarity and induced under frost stress. It has been suggested that the members of the $C b f$ gene family have slightly different functions in the frost responsive network [8,9]. In this study, a large variation of mean $r^{2}$ in seven $C b f$ genes (0.13 to 0.92) was observed, indicating that the family has probably undergone diverse selection history. LD can be 
increased by selection, for instance, by selective sweeps in which the alleles at flanking loci of a locus under selection are rapidly swept to high frequency or fixation [70]. Arabidopsis' AtCbf2 was implicated as subject to selection, resulting in functional divergence from AtCbf1 and $A t C b f 3$ after $C b f$ gene duplication [54]. In this study an observed strong LD block and low nucleotide diversity in $\mathrm{ScCbf14}$ indicated a selective sweep. Among Cbf family members, $\mathrm{TaCbf} 14$ has been mapped to the highest peak of the frost tolerance QTL in hexaploid wheat [7]. Two of the SNPs in $H v C b f 14$ were statistically associated with frost tolerance in a European germplasm collection of spring and winter barley [36]. It remains to be demonstrated that the LD block of ScCbf14 found in this study has an influence on frost tolerance in rye.

\section{Conclusions}

Genetic diversity is vital to crop improvement. This study of eleven candidate genes with a putative role in frost response and 37 genome-wide SSRs demonstrated high genetic diversity among five winter rye populations from Middle and Eastern Europe. Most of the diversity was observed within populations. The Middle European Petkus population differed neither in terms of haplotype frequencies nor in nucleotide diversities in eleven candidate genes from the four Eastern European populations. LD within candidate genes decayed rapidly, falling below $r^{2}=0.16$ within approximately $520 \mathrm{bp}$. In contrast to selfing species, such as Arabidopsis or barley, low LD in rye promises a higher resolution in genome-wide association mapping. A challenge, however, is that many more markers are required for covering the whole genome. Given the huge genome size of rye, $(\sim 8,100 \mathrm{Mb})$ and until high-density genotyping arrays for rye become available, candidate gene based association mapping remains the most appropriate strategy for gene identification. The SNPs discovered in the promoter or coding regions of the genes investigated in this study, which cause non-synonymous substitutions, are suitable candidates for association mapping and will be studied in more detail with respect to their role in the expression of frost tolerance in rye.

\section{Additional material}

Additional file 1: Primer information and details on PCR amplification of eleven candidate genes.

Additional file 2: Genetic diversities of eleven candidate genes within five rye populations.

Additional file 3: Chromosomal locations and diversities of the 37 SSRs.

Additional file 4: Analysis of molecular variance (AMOVA) based on 37 SSR markers.

Additional file 5: Scatterplots of pairwise distances and LD.

\section{Acknowledgements}

We would like to thank Susanne Schrack and Tobias Dreser for technical assistance and Carmen Berlanas for sequencing ScCbf6 in her master thesis. We acknowledge Andreas Böck and Valentin Wimmer for their help in determining the LD extent. We thank the two anonymous reviewers for their constructive comments. The first author gratefully acknowledges the support of the Graduate School at the Technische Universität München, München, Germany. The project GABI RYE-FROST is funded by the German Federal Ministry of Education and Research (Grant numbers 0315062A and 0315062B).

\section{Author details}

${ }^{1}$ Technische Universität München, Plant Breeding, Freising, Germany. ${ }^{2}$ Technische Universität München, Mathematical Statistics, Garching, Germany. ${ }^{3}$ KWS LOCHOW GMBH, Bergen, Germany.

\section{Authors' contributions}

YL carried out the candidate gene and statistical analyses and drafted the manuscript. GH participated in the molecular and statistical analyses. DA provided advice for the statistical analysis. VK provided SSR marker data. PW developed the plant material. EB, CCS, PW, and VK conceived the study. All authors read, edited and approved the final manuscript.

Received: 12 August 2010 Accepted: 10 January 2011

Published: 10 January 2011

\section{References}

1. Fowler DB, Limin AE: Exploitable genetic variability for cold tolerance in commercially grown cereals. Can J Plant Sci 1987, 67(1):278-278.

2. Thomashow MF: Plant cold acclimation: Freezing tolerance genes and regulatory mechanisms. Annu Rev Plant Physiol Plant Mol Biol 1999, 50:571-599.

3. Yamaguchi-Shinozaki K, Shinozaki K: Transcriptional regulatory networks in cellular responses and tolerance to dehydration and cold stresses. Annu Rev Plant Biol 2006, 57:781-803.

4. Badawi M, Reddy YV, Agharbaoui Z, Tominaga Y, Danyluk J, Sarhan F, Houde M: Structure and functional analysis of wheat ICE (inducer of CBF expression) genes. Plant Cell Physiol 2008, 49(8):1237-1249.

5. Fursova OV, Pogorelko GV, Tarasov VA: Identification of ICE2, a gene involved in cold acclimation which determines freezing tolerance in Arabidopsis thaliana. Gene 2009, 429(1-2):98-103.

6. Francia E, Barabaschi D, Tondelli A, Laido G, Rizza F, Stanca AM, Busconi M, Fogher C, Stockinger EJ, Pecchioni N: Fine mapping of a HvCBF gene cluster at the frost resistance locus Fr-H2 in barley. Theor Appl Genet 2007, 115(8):1083-1091.

7. Baga M, Chodaparambil SV, Limin AE, Pecar M, Fowler DB, Chibbar RN: Identification of quantitative trait loci and associated candidate genes for low-temperature tolerance in cold-hardy winter wheat. Funct Integr Genomics 2007, 7(1):53-68.

8. Knox AK, Li CX, Vagujfalvi A, Galilba G, Stockinger EJ, Dubcovsky J: Identification of candidate CBF genes for the frost tolerance locus Fr-A (m)2 in Triticum monococcum. Plant Mol Biol 2008, 67(3):257-270.

9. Campoli C, Matus-Cadiz MA, Pozniak CJ, Cattivelli L, Fowler DB: Comparative expression of $\mathrm{Cbf}$ genes in the Triticeae under different acclimation induction temperatures. Mol Genet Genomics 2009, 282(2):141-152.

10. Alonso-Blanco C, Gomez-Mena C, Llorente F, Koornneef M, Salinas J, Martinez-Zapater JM: Genetic and molecular analyses of natural variation indicate CBF2 as a candidate gene for underlying a freezing tolerance quantitative trait locus in Arabidopsis. Plant Physiol 2005, 139(3):1304-1312.

11. McKhann HI, Gery C, Berard A, Leveque S, Zuther E, Hincha DK, De Mita S, Brunel $D$, Teoule E: Natural variation in CBF gene sequence, gene expression and freezing tolerance in the Versailles core collection of Arabidopsis thaliana. BMC Plant Biol 2008, 8:105.

12. Chinnusamy V, Zhu J, Zhu JK: Cold stress regulation of gene expression in plants. Trends Plant Sci 2007, 12(10):444-451.

13. Kosova $K$, Vitamvas P, Prasil IT: The role of dehydrins in plant response to cold. Biol Plant 2007, 51(4):601-617.

14. Choi DW, Zhu B, Close TJ: The barley (Hordeum vulgare L.) dehydrin multigene family: sequences, allele types, chromosome assignments, 
and expression characteristics of 11 Dhn genes of cv Dicktoo. Theor Appl Genet 1999, 98(8):1234-1247.

15. Zhu B, Choi DW, Fenton R, Close TJ: Expression of the barley dehydrin multigene family and the development of freezing tolerance. Mol Gen Genet 2000, 264(1-2):145-153

16. Kosova K, Prasil IT, Vitamvas P: The relationship between vernalization-and photoperiodically-regulated genes and the development of frost tolerance in wheat and barley. Biol Plant 2008, 52(4):601-615.

17. Galiba G, Vagujfalvi A, Li CX, Soltesz A, Dubcovsky J: Regulatory genes involved in the determination of frost tolerance in temperate cereals. Plant Sci 2009, 176(1):12-19.

18. Galiba G, Quarrie SA, Sutka J, Morgounov A, Snape JW: RFLP mapping of the vernalization $(V r n 1)$ and frost resistance ( $F r 1)$ genes on chromosome 5A of wheat. Theor Appl Genet 1995, 90(7-8):1174-1179.

19. Stockinger EJ, Skinner JS, Gardner KG, Francia E, Pecchioni N: Expression levels of barley $\mathrm{Cbf}$ genes at the Frost resistance- $\mathrm{H} 2$ locus are dependent upon alleles at Fr-H1 and Fr-H2. Plant J 2007, 51(2):308-321.

20. Persson $K$, von Bothmer R, Gullord M, Gunnarsson E: Phenotypic variation and relationships in landraces and improved varieties of rye (Secale cereale $L$.) from northern Europe. Genet Resour Crop Evol 2006, 53(4):857-866.

21. Persson K, Von Bothmer R: Genetic diversity amongst landraces of rye (Secale cereale L.) from northern Europe. Hereditas 2002, 136(1):29-38.

22. Shang HY, Wei YM, Wang XR, Zheng YL: Genetic diversity and phylogenetic relationships in the rye genus Secale $L$. (rye) based on Secale cereale microsatellite markers. Genet Mol Biol 2006, 29(4):685-691.

23. Flint-Garcia SA, Thornsberry JM, Buckler ES: Structure of linkage disequilibrium in plants. Annu Rev Plant Biol 2003, 54:357-374.

24. Maccaferri M, Sanguineti MC, Noli E, Tuberosa R: Population structure and long-range linkage disequilibrium in a durum wheat elite collection. $\mathrm{Mol}$ Breed 2005, 15(3):271-289.

25. Tenaillon MI, Sawkins MC, Long AD, Gaut RL, Doebley JF, Gaut BS: Patterns of DNA sequence polymorphism along chromosome 1 of maize (Zea mays ssp mays L.). Proc Natl Acad Sci USA 2001, 98(16):9161-9166.

26. Ching A, Caldwell KS, Jung M, Dolan M, Smith OS, Tingey S, Morgante M, Rafalski AJ: SNP frequency, haplotype structure and linkage disequilibrium in elite maize inbred lines. BMC Genet 2002, 3:19.

27. Hamblin MT, Fernandez MGS, Casa AM, Mitchell SE, Paterson AH, Kresovich S: Equilibrium processes cannot explain high levels of shortand medium-range linkage disequilibrium in the domesticated grass Sorghum bicolor. Genetics 2005, 171(3):1247-1256.

28. Caldwell KS, Russell J, Langridge P, Powell W: Extreme populationdependent linkage disequilibrium detected in an inbreeding plant species, Hordeum vulgare. Genetics 2006, 172(1):557-567.

29. Rogowsky PM, Guidet FLY, Langridge P, Shepherd KW, Koebner RMD: Isolation and characterisation of wheat-rye recombinants involving chromosome arm 1DS of wheat. Theor Appl Genet 1991, 82(5):537-544.

30. Campoli C, Matus-Cadiz MA, Pozniak CJ, Cattivelli L, Fowler DB: Comparative expression of $\mathrm{Cbf}$ genes in the Triticeae under different acclimation induction temperatures. Molecular Genetics and Genomics 2009, 282(2):141-152.

31. Sutton F, Chen DG, Ge XJ, Kenefick D: Cbf genes of the Fr-A2 allele are differentially regulated between long-term cold acclimated crown tissue of freeze-resistant and - susceptible, winter wheat mutant lines. BMC Plant Biol 2009, 9:34.

32. Skinner JS, von Zitzewitz J, Szucs P, Marquez-Cedillo L, Filichkin T, Amundsen $\mathrm{K}$, Stockinger EJ, Thomashow MF, Chen THH, Hayes PM: Structural, functional, and phylogenetic characterization of a large CBF gene family in barley. Plant Mol Biol 2005, 59(4):533-551.

33. Jaglo KR, Kleff S, Amundsen KL, Zhang X, Haake V, Zhang JZ, Deits T, Thomashow MF: Components of the Arabidopsis C-repeat/dehydrationresponsive element binding factor cold-response pathway are conserved in Brassica napus and other plant species. Plant Physiol 2001, 127(3):910-917.

34. Beales J, Laurie DA, Devos KM: Allelic variation at the linked AP1 and PhyC loci in hexaploid wheat is associated but not perfectly correlated with vernalization response. Theor Appl Genet 2005, 110(6):1099-1107.

35. Yan L, Loukoianov A, Tranquilli G, Helquera M, Fahima T, Dubcovsky J: Positional cloning of the wheat vernalization gene VRN1. Proc Natl Acad Sci USA 2003, 100(10):6263-6268.

36. Fricano A, Rizza F, Faccioli P, Pagani D, Pavan P, Stella A, Rossini L, Piffanelli P, Cattivelli L: Genetic variants of HvCbf14 are statistically associated with frost tolerance in a European germplasm collection of Hordeum vulgare. Theoretical and Applied Genetics 2009, 119(7):1335-1348

37. Knox AK, Dhillon T, Cheng HM, Tondelli A, Pecchioni N, Stockinger EJ: CBF gene copy number variation at Frost Resistance-2 is associated with levels of freezing tolerance in temperate-climate cereals. Theor Appl Genet 2010, 121(1):21-35.

38. Yang ZH: PAML 4: Phylogenetic analysis by maximum likelihood. Mol Biol Evol 2007, 24(8):1586-1591.

39. Khlestkina EK, Ma HMT, Pestsova EG, Roder MS, Malyshev SV, Korzun V, Borner A: Mapping of 99 new microsatellite-derived loci in rye (Secale cereale L.) including 39 expressed sequence tags. Theor Appl Genet 2004, 109(4):725-732.

40. Hackauf $B$, Wehling P: Identification of microsatellite polymorphisms in an expressed portion of the rye genome. Plant Breed 2002, 121(1):17-25.

41. Liu KJ, Muse SV: PowerMarker: an integrated analysis environment for genetic marker analysis. Bioinformatics 2005, 21(9):2128-2129.

42. Petit RJ, El Mousadik A, Pons O: Identifying populations for conservation on the basis of genetic markers. Conserv Biol 1998, 12(4):844-855.

43. Goudet J: FSTAT (Version 1.2): A computer program to calculate Fstatistics. J Hered 1995, 86(6):485-486.

44. Dice LR: Measure of the amount of ecologic association between species. Ecology 1945, 26(3):297-302.

45. Excoffier L, Smouse PE, Quattro JM: Analysis of molecular variance inferred from metric distances among DNA haplotypes: application to human mitochondrial DNA restriction data. Genetics 1992, 131(2):479-491.

46. Excoffier L, Laval G, Schneider S: Arlequin (version 3.0): An integrated software package for population genetics data analysis. Evolutionary Bioinformatics 2005, 1:47-50.

47. Hill WG, Robertson A: Linkage disequilibrium in finite populations. Theor Appl Genet 1968, 38:226-231.

48. Weir B: Genetic data analysis II. Sunderland, MA: Sinauer Associates; 1996.

49. Hill WG, Weir BS: Variances and covariance of squared linkage disequilibrium in finite populations. Theor Popul Biol 1988, 33(1):54-78.

50. Breseghello F, Sorrells ME: Association mapping of kernel size and milling quality in wheat (Triticum aestivum L.) cultivars. Genetics 2006, 172(2):1165-1177.

51. Nei M: Molecular Evolutionary Genetics. NY: Columbia University Press; 1987.

52. Yamasaki M, Schroeder S, Sanchez-Villeda H, Gaut B, McMullen MD: Empirical analysis of selection screens for domestication and improvement loci in maize by extended DNA sequencing. Plant Genome 2008, 1(1):33-43

53. Krutovsky KV, Neale DB: Nucleotide diversity and linkage disequilibrium in cold-hardiness- and wood quality-related candidate genes in Douglas fir. Genetics 2005, 171(4):2029-2041.

54. Lin YH, Hwang SY, Hsu PY, Chiang YC, Huang CL, Wang CN, Lin TP: Molecular population genetics and gene expression analysis of duplicated CBF genes of Arabidopsis thaliana. BMC Plant Biol 2008, 8:16.

55. Lawton-Rauh A: Evolutionary dynamics of duplicated genes in plants. Mol Phylogenet Evol 2003, 29(3):396-409.

56. Biswas S, Akey JM: Genomic insights into positive selection. Trends Genet 2006, 22(8):437-446.

57. Wachowiak W, Balk PA, Savolainen O: Search for nucleotide diversity patterns of local adaptation in dehydrins and other cold-related candidate genes in Scots pine (Pinus sylvestris L.). Tree Genet Genomes 2009, 5(1):117-132

58. Yang Z: Adaptive molecular evolution. In Handbook of Statistical Genetics. Volume 1. Edited by: Balding DJ, Bishop M, Cannings C. West Sussex: John Wiley 2003:229-250.

59. Kryazhimskiy S, Plotkin JB: The Population Genetics of $d N / d S$. Plos Genet 2008, 4(12):e1000304.

60. Kilian B, Ozkan H, Kohl J, von Haeseler A, Barale F, Deusch O, Brandolini A, Yucel C, Martin W, Salamini F: Haplotype structure at seven barley genes: relevance to gene pool bottlenecks, phylogeny of ear type and site of barley domestication. Mol Genet Genomics 2006, 276(3):230-241.

61. Tian F, Stevens NM, Buckler ES: Tracking footprints of maize domestication and evidence for a massive selective sweep on chromosome 10. Proc Natl Acad Sci USA 2009, 106:9979-9986.

62. George J, Dobrowolski MP, de Jong EV, Cogan NOI, Smith KF, Forster JW: Assessment of genetic diversity in cultivars of white clover (Trifolium repens L.) detected by SSR polymorphisms. Genome 2006, 49(8):919-930. 
63. Bolaric S, Barth S, Melchinger AE, Posselt UK: Genetic diversity in European perennial ryegrass cultivars investigated with RAPD markers. Plant Breed 2005, 124(2):161-166.

64. Zhang DL, Zhang HL, Wang MX, Sun JL, Qi YW, Wang FM, Wei XH, Han LZ, Wang XK, Li ZC: Genetic structure and differentiation of Oryza sativa L. in China revealed by microsatellites. Theor Appl Genet 2009, 119(6):1105-1117.

65. Xing Y, Frei U, Schejbel B, Asp T, Lubberstedt T: Nucleotide diversity and linkage disequilibrium in 11 expressed resistance candidate genes in Lolium perenne. BMC Plant Biol 2007, 7:43.

66. Kim S: Recombination and linkage disequilibrium in Arabidopsis thaliana. Nat Genet 2007, 39:1151-1155.

67. Nordborg M, Hu TT, Ishino Y, Jhaveri J, Toomajian C, Zheng HG, Bakker E, Calabrese P, Gladstone J, Goyal R, Jakobsson M, Kim S, Morozov Y Padhukasahasram B, Plagnol V, Rosenberg NA, Shah C, Wall JD, Wang J, Zhao KY, Kalbfleisch T, Schulz V, Kreitman M, Bergelson J: The pattern of polymorphism in Arabidopsis thaliana. PLoS Biol 2005, 3(7):1289-1299.

68. Gustafson JP, Ma XF, Korzun V, Snape JW: A consensus map of rye integrating mapping data from five mapping populations. Theor Appl Genet 2009, 118(4):793-800

69. Badawi M, Danyluk J, Boucho B, Houde M, Sarhan F: The CBF gene family in hexaploid wheat and its relationship to the phylogenetic complexity of cereal CBFs. Mol Genet Genomics 2007, 277(5):533-554.

70. Kraakman ATW, Niks RE, Van den Berg P, Stam P, Van Eeuwijk FA: Linkage disequilibrium mapping of yield and yield stability in modern spring barley cultivars. Genetics 2004, 168(1):435-446.

doi:10.1186/1471-2229-11-6

Cite this article as: Li et al:: High levels of nucleotide diversity and fast decline of linkage disequilibrium in rye (Secale cereale L.) genes involved in frost response. BMC Plant Biology 2011 11:6.

\section{Submit your next manuscript to BioMed Central} and take full advantage of:

- Convenient online submission

- Thorough peer review

- No space constraints or color figure charges

- Immediate publication on acceptance

- Inclusion in PubMed, CAS, Scopus and Google Scholar

- Research which is freely available for redistribution

Submit your manuscript at www.biomedcentral.com/submit
Biomed Central 